\title{
Blue and white light emission from zinc oxide nanoforests
}

\author{
Nafisa Noor ${ }^{\star}, \ddagger 1$, Luca Lucera ${ }^{\ddagger 2, \S}$, Thomas Capuano ${ }^{3, \S}$, Venkata Manthina ${ }^{4, \pi}$, \\ Alexander G. Agrios ${ }^{5,6}$, Helena Silva ${ }^{1}$ and Ali Gokirmak ${ }^{1}$
}

\section{Full Research Paper}

Address:

${ }^{1}$ Department of Electrical and Computer Engineering, University of Connecticut, Storrs, Connecticut 06269, USA, ${ }^{2}$ ZAE Bayern - Solar Factory of the Future, Fürtherstrasse 250, 90429 Nürnberg, Germany, ${ }^{3}$ National Instruments, Austin, Texas 78759, USA, ${ }^{4}$ Fraunhofer Center for Energy Innovation (CEI), University of Connecticut, Storrs, Connecticut 06269, USA, ${ }^{5}$ Department of Civil and Environmental Engineering, University of Connecticut, Storrs, Connecticut 06269, USA, and ${ }^{6}$ Center for Clean Energy Engineering, University of Connecticut, Storrs, Connecticut 06269, USA

Email:

Nafisa Noor ${ }^{*}$ - nafisa.noor@engr.uconn.edu

* Corresponding author $\ddagger$ Equal contributors

$\S$ This research was performed while L. Lucera and T. Capuano were with the Department of Electrical and Computer Engineering, University of Connecticut.

II This research was performed while V. Manthina was with the Department of Civil and Environmental Engineering, University of Connecticut.

Keywords:

electrical stress; light emission; nanoforest; plasma; zinc oxide
Beilstein J. Nanotechnol. 2015, 6, 2463-2469.

doi:10.3762/bjnano.6.255

Received: 15 September 2015

Accepted: 04 December 2015

Published: 23 December 2015

Associate Editor: P. Leiderer

(C) 2015 Noor et al; licensee Beilstein-Institut. License and terms: see end of document.

\begin{abstract}
Blue and white light emission is observed when high voltage stress is applied using micrometer-separated tungsten probes across a nanoforest formed of $\mathrm{ZnO}$ nanorods. The optical spectrum of the emitted light consistently shows three fine peaks with very high amplitude in the 465-485 nm (blue) range, corresponding to atomic transitions of zinc. Additional peaks with smaller amplitudes in the $330-650 \mathrm{~nm}$ range and broad spectrum white light is observed depending on the excitation conditions. The spatial and spectral distribution of the emitted light, with pink-orange regions identifying percolation paths in some cases and high intensity blue and white light with center to edge variations in others, indicate that multiple mechanisms lead to light emission. Under certain conditions, the tungsten probe tips used to make electrical contact with the $\mathrm{ZnO}$ structures melt during the excitation, indicating that the local temperature can exceed $3422{ }^{\circ} \mathrm{C}$, which is the melting temperature of tungsten. The distinct and narrow peaks in the optical spectra and the abrupt increase in current at high electric fields suggest that a plasma is formed by application of the electrical bias, giving rise to light emission via atomic transitions in gaseous zinc and oxygen. The broad spectrum, white light emission is possibly due to the free electron transitions in the plasma and blackbody radiation from molten silicon. The white light may also arise from the recombination through multiple defect levels in $\mathrm{ZnO}$ or due to the optical excitation from solid $\mathrm{ZnO}$. The electrical measurements performed at different ambient pressures result in light emission with distinguishable differences in the emission properties
\end{abstract}


and $I-V$ curves, which also indicate that the dielectric breakdown of $\mathrm{ZnO}$, sublimation, and plasma formation processes are the underlying mechanisms.

\section{Introduction}

The interest in environmentally friendly semiconductors, biocompatible [1], functional nanostructures [2], nanoscale electronic devices and large area electronics has led to significant research efforts in metal-oxide semiconductors such as $\mathrm{ZnO} . \mathrm{ZnO}$ is a common, low-cost, antibacterial [3] material that forms various nanostructures depending on the process conditions. It is a direct and wide band gap semiconductor $(\approx 3.4 \mathrm{eV})$ with large exciton binding energy $(60 \mathrm{meV})$. $\mathrm{ZnO}$ nanowires have been shown to yield stimulated emission with optical pumping (e.g., nanowire laser) [4] and have been demonstrated as photodetectors [5]. ZnO films have also been used in transparent thin film transistors [6] and as phosphors [7]. ZnO typically grows as an n-type semiconductor due to oxygen deficiencies and has been used as the n-type material in heterojunctions [8], but there have also been significant efforts to produce highquality p-type $\mathrm{ZnO}$ to form homojunction light emitting diodes (LED) that can produce UV [9], blue [10] or white light [11]. Recently there have also been reports on dye-sensitized solar cells $[12,13]$ that utilize $\mathrm{ZnO}$ nanostructures. $\mathrm{ZnO}$, with its interesting electronic and optical properties [14] and possibility of synthesis using relatively simple approaches, can become a low-cost alternative to $\mathrm{GaN}$ [8]. It is also one of the substances that sublimate congruently at atmospheric pressure [15-17]:

$$
\mathrm{ZnO}(s) \rightarrow \mathrm{Zn}(g)+\frac{1}{2} \mathrm{O}_{2}(g)
$$

The results we present here are from electrical experiments performed on $\mathrm{ZnO}$ nanoforests formed by high-density nanowires grown on highly doped silicon microstructures. We have observed light emission upon application of relatively high electric fields $(\approx 3-7 \mathrm{~V} / \mu \mathrm{m})$ to the nanoforests using tungsten probes. We have performed electrical characterization at different ambient pressures and analyzed the resulting optical emission spectra and videos to determine the mechanisms giving rise to light emission.

\section{Experimental $\mathrm{ZnO}$ growth}

Chemical bath deposition (CBD), a low-cost solution-based technique $[18,19]$, is used to grow $\mathrm{ZnO}$ nanorods on oxidized silicon wafers with previously fabricated, highly doped, p-type, nanocrystalline silicon microstructures. The samples were precleaned by sonication in ethanol, dried with nitrogen and spin-coated with a seed solution that was prepared by dissolving $0.0457 \mathrm{mg}$ of zinc acetate dihydrate in $50 \mathrm{~mL}$ of ethanol. The samples were then baked at $350{ }^{\circ} \mathrm{C}$ for $30 \mathrm{~min}$ and then submersed in a water-based precursor solution containing $25 \mathrm{mM}$ of zinc nitrate hexahydrate, $25 \mathrm{mM}$ of hexamethylenetetramine and $6 \mathrm{mM}$ of poly(ethyleneimine) for $24 \mathrm{~h}$, and kept at $90{ }^{\circ} \mathrm{C}$. The process yields $2-2.5 \mu \mathrm{m}$ long $\mathrm{ZnO}$ nanorods homogeneously grown along the $c$-direction of the wurtzite structure with 50-250 nm diameter (Figure 1) on top of a very thin layer of $\mathrm{ZnO}$ film $(\approx 2-3 \mathrm{~nm})$.

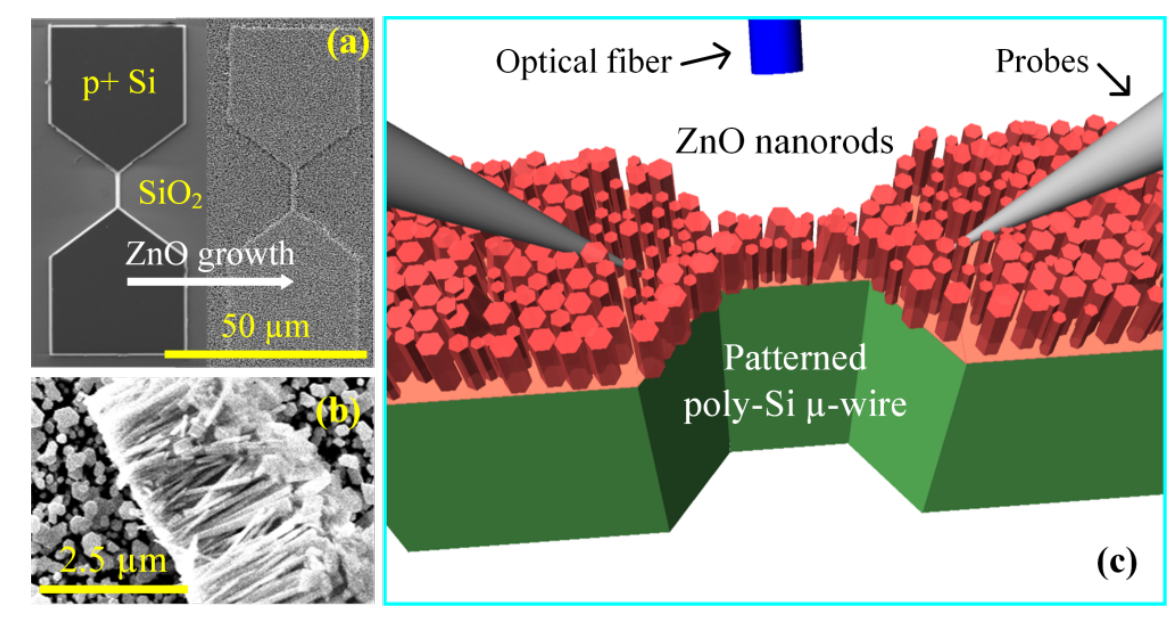

Figure 1: (a) SEM image of the ZnO nanorods grown on $\approx 100 \mathrm{~nm}$ thick, highly doped, patterned, p-type, silicon microstructures (before and after ZnO nanorod growth), (b) cross-section SEM image of the ZnO nanorods, and (c) schematic of the electrical probe and optical fiber arrangement. 


\section{Experimental setup}

DC voltage sweeps between $0-80 \mathrm{~V}$, AC sinusoidal voltages with peak-to-peak amplitudes up to $80 \mathrm{~V}$, and $1-10 \mathrm{kHz}$ frequency and microsecond single pulses with amplitudes up to $50 \mathrm{~V}$ were applied to the samples in open air and in vacuum in a probe station, which was equipped with high-magnification optics and high-resolution micromanipulators. The DC, AC, and pulse excitations were applied using a HP 4145B semiconductor parameter analyzer, a Tektronix AFG 3102 arbitrary/ function generator, and an Agilent 8114A pulse generator, respectively. The atmospheric pressure measurements were performed in an Alessi probe station and those in vacuum were done in a Janis cryogenic probe station at 2.5 mTorr (medium vacuum).

The tungsten probe tips with $2.4 \mu \mathrm{m}$ tip radius and $45^{\circ}$ angle (Cascade Microtech, PTT-24/4-25) were forced to slightly slide through the $\mathrm{ZnO}$ nanoforest in order to ensure good electrical contact (Figure 1c). The probe separation for all electrical measurements was $\approx 10-15 \mu \mathrm{m}$. Either a high-sensitivity $1080 \mathrm{p}$ HD camcorder with a frame rate of 60 fps (Sony, HDR-CX160) or a high-speed camera with a maximum of $1200 \mathrm{fps}$ (Casio, EX-F1) was connected to the microscope head for imaging during the measurements. In order to understand the physical mechanisms that lead to light emission, a wideband (200-1100 nm) optical spectrometer with $1 \mathrm{~nm}$ resolution (Ocean Optics, HR2000+) coupled with an optical fiber was incorporated with the system for spectral analysis. The fiber tip was attached to a probe arm and was aligned to the test area using a micromanipulator.

A high-speed PIN diode with built-in amplifier was attached to another micromanipulator and was positioned to face the test area in order to detect the emitted light intensity with better time resolution. The distance from the PIN diode to the sample was adjusted to achieve adequate signal-to-noise ratio without saturating the diode during the experiments. The voltage output of the built-in amplifier was significantly larger than any noise or perturbations coupled into the signal lines caused by the fast pulses. Hence, this approach was preferred over a simple PIN diode for detection. The applied voltage, PIN diode output voltage, and resulting current were measured simultaneously using two synchronized two-channel oscilloscopes (Tektronix, TDS 2002B) for the AC and pulse measurements.

The coaxial cable lengths were matched to minimize the difference in phase delay. The output of the signal generator and the inputs to the oscilloscopes were terminated to $50 \Omega$ resistors to eliminate reflections and oscillations (Figure 2). A black enclosure surrounding the whole setup was used and the other room light sources were turned off during the measurements to elimi-

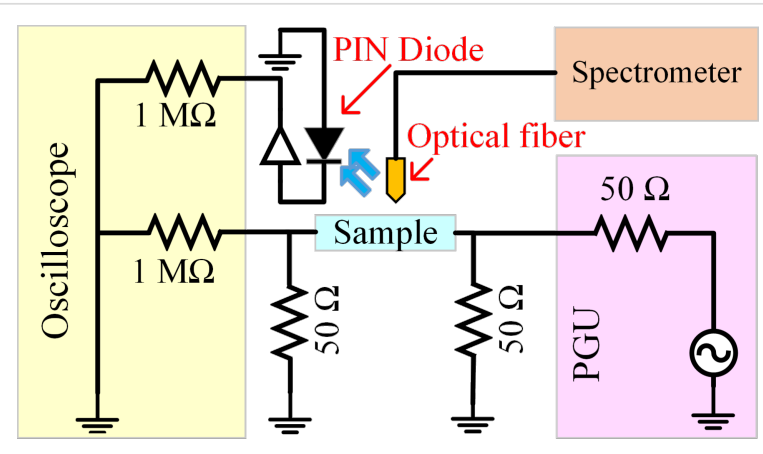

Figure 2: The schematics of a typical measurement setup with a pulse generating unit (PGU). An optical spectrometer was used to perform spectral analysis with time resolution of $\geq 1 \mathrm{~ms}$ in the $200-1100 \mathrm{~nm}$ range.

nate ambient light. The measurements were controlled through a computer.

The electrical resistivities of the bare polysilicon substrate and the sample with $\mathrm{ZnO}$ nanorods on polysilicon were measured using a four-point probe method.

\section{Results and Discussion}

In all experiments, bright blue and white light and high current levels were observed as the voltage level was increased (Figure 3 and Figure 4). In some cases orange-pink regions were also observed (Figure 3 and Figure 4 bottom-right). The light emission was observed to be very intense, scaling with the applied voltage and observed as multiple, brief flashes during long excitations. The emitted light seemed to follow a changing percolation path between the contacts (Figure 3, multimedia view) [20] as the materials experience changes in thermal and electrical properties over time due to the high electrical stress.

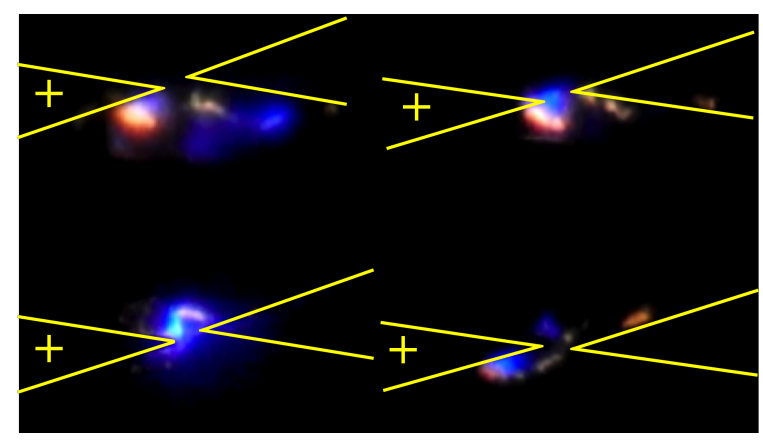

Figure 3: Frames extracted from high-speed videos showing light emission and changing percolation paths during DC tests with an approximate probe distance of $10-15 \mu \mathrm{m}$. The probe locations and applied voltage polarity are as indicated in yellow (multimedia view).

Since the $\mathrm{ZnO}$ nanoforest material has relatively weak mechanical properties and adhesion to the substrate, probing with tung- 

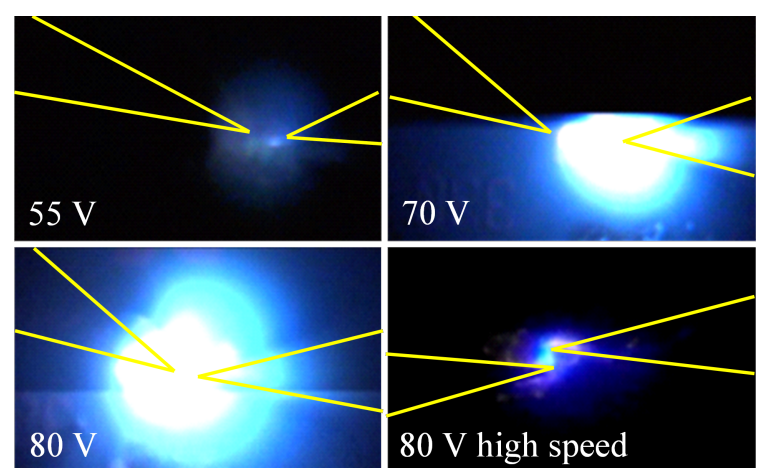

Figure 4: Frames extracted from high-resolution videos showing light emission from the $\mathrm{ZnO}$ nanorods contacted by tungsten probes during AC excitation of $10-10000 \mathrm{~Hz}$ with the indicated peak-to-peak voltage level. The approximate probe separation was $10-15 \mu \mathrm{m}$. Similar visible emissions occurred with pulsed excitation. The probe positions are as indicated in yellow.

sten tips causes some shifting of this material and so the probes are likely to touch both $\mathrm{ZnO}$ and the polysilicon substrate. The room-temperature electrical resistivity of the $\mathrm{ZnO}$ nanoforest on highly doped polysilicon is $\approx 6.8 \Omega \cdot \mathrm{cm}$ and that of the highly doped polysilicon substrate itself is $\approx 0.02 \Omega \cdot \mathrm{cm}$. Therefore, the current is expected to mostly flow through the polysilicon substrate, at least in the beginning when the temperature is low. As the temperature increases, due to Joule heating, the current paths will change depending on the temperature dependence of the resistivity of these two materials. Due to the large difference in resistivity, however, it is likely that the current flows mostly through the polysilicon substrate until the electrical breakdown of air or the $\mathrm{ZnO}$ nanorods occurs under the high fields and lower resistivity paths open through the $\mathrm{ZnO}$ nanoforest.

Because of the geometrical nonuniformity of the $\mathrm{ZnO}$ nanoforest, the electrical resistance and electric field distribution between two probes are also nonuniform. Therefore, some segments along the current flow path are likely to undergo dielectric breakdown and joule heating earlier than others. The joule heating in turn results in sublimation of $\mathrm{ZnO}$ into $\mathrm{Zn}$ vapor and $\mathrm{O}_{2}$ gas and melting of the polysilicon substrate. Thus different regions between the two probes are expected to experience these thermal changes in a sequential fashion.

Typically $3.4 \mathrm{eV}$ emission (UV light) is expected from $\mathrm{ZnO}$ if conduction band to valance band recombination is the dominant mechanism. White light can also be emitted from the $\mathrm{ZnO}$ structures as the excited carriers trickle down from the conduction band to valance band through multiple defect levels or because of the optical excitation of materials by the ultraviolet source [21]. Blue light has been previously observed from $\mathrm{ZnO}$-based homojunction LEDs, where it is attributed to donor-acceptor pair recombination in the p-type $\mathrm{ZnO}$ layer [10] and also from $\mathrm{n}-\mathrm{ZnO} / \mathrm{p}-\mathrm{GaN}$ heterojunction LEDs [22]. The superposition of electroluminescence and Fabry-Pérot oscillations has also been suggested as the possible mechanism for blue-white emission from $\mathrm{ZnO}$-based homojunction LEDs [23].

The spectra obtained in this work (from a large number of measurements with DC, AC and pulse analyses) consistently showed three, high intensity, fine spectral lines at approximately 481, 472 and $467 \mathrm{~nm}$ (Figure 5a). This triplet (in blue
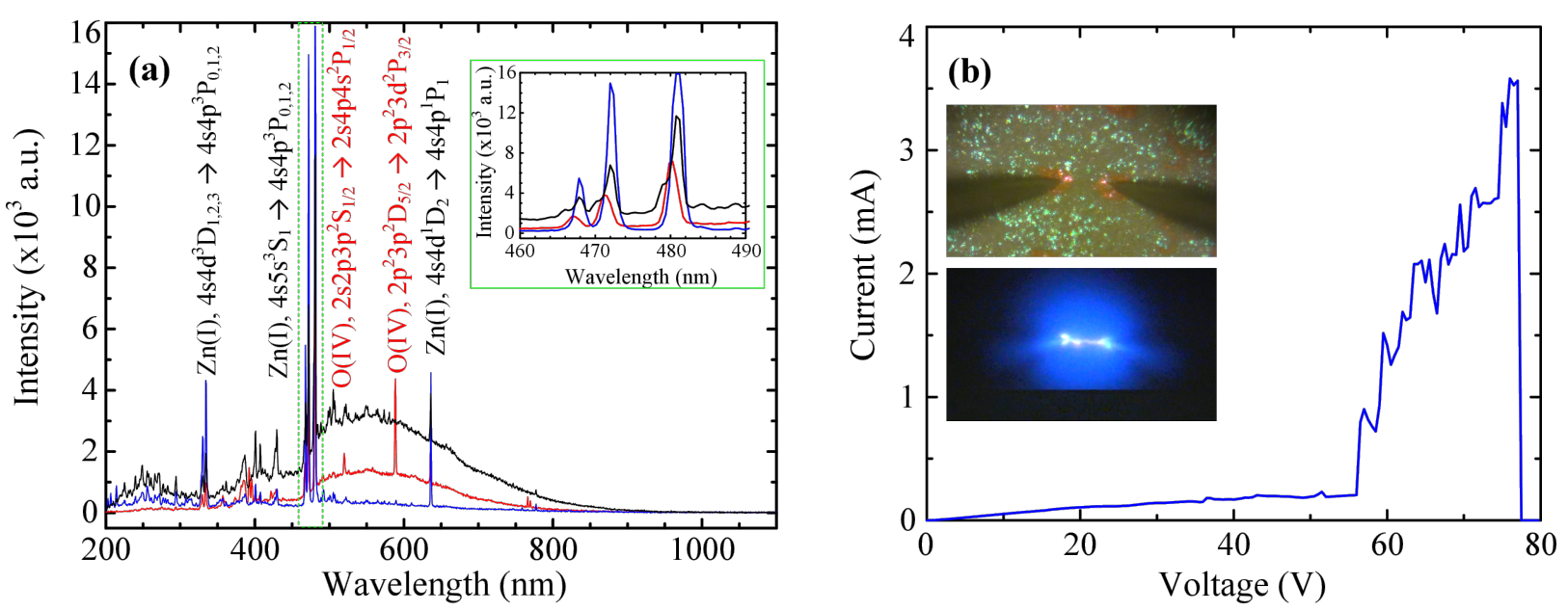

Figure 5: (a) Optical emission spectra obtained from DC voltage sweeping of 0-80 V (blue), AC excitation with an amplitude of $40 \mathrm{~V}$ at $1 \mathrm{kHz}$ (red), and single pulse voltage with an amplitude of $35 \mathrm{~V}$ and a duration of $10 \mu \mathrm{s}$ (black). The corresponding atomic electron transitions in the excited species are indicated adjacent to the spectral lines. The inset shows the characteristic peaks (triplet) for neutral $\mathrm{Zn}$ atoms. (b) Example of a DC, $I-V$ characteristic from $0-80 \mathrm{~V}$ in steps of $1 \mathrm{~V}$ through the $\mathrm{ZnO}$ nanorods. The entire DC sweep was carried out in $\approx 10-15 \mathrm{~s}$. The insets show the optical image of the probe arrangement before the test (top) and a frame from the high-resolution video of the light emission (bottom). 
wavelength range in Figure 5a inset) corresponds to atomic electron transitions (AETs) in neutral zinc (Zn I) atoms. The localized orange-pink glow observed in some cases $(\approx 635 \mathrm{~nm})$ can also be ascribed to another AET of neutral zinc (Figure 5). Hence, the existence of these fine lines indicates generation of a plasma by the dielectric breakdown of air between the $\mathrm{ZnO}$ nanorods and of the breakdown of the $\mathrm{ZnO}$ structures themselves, leading to sublimation and plasma formation in the $\mathrm{ZnO}$ nanoforest.

The application of higher amplitude and longer duration voltage pulses resulted in a greater overall intensity of the emitted light (Figure 5, Figure 6). The broad spectrum (white light) emission (Figure 5 and Figure 6) can be ascribed to the free-free electron transitions in the plasma, the electronic excitations within solid $\mathrm{ZnO}$ and/or energy loss through multiple transitions with a very broad spectrum of trap levels [24]. Blackbody radiation may also contribute to the broad spectrum, white light emission but the spectral distribution is distinctly different than what is expected from blackbody radiation alone. The lack of broad spectrum emission in some of the DC experiments indicates a distinct difference in plasma conditions (Figure 5). The intensity of the emitted light was often strong enough to saturate the spectrum analyzer, PIN diode and the camera, even for a single $\mu$ s-duration pulse (Figure 6a). The pulse measurements showed a nonsteady and high current level (Figure 6b).

The SEM images taken after DC electrical analysis (Figure 7) indicate melting of the materials between the contacts, and an optical image of the tungsten probes after some of the measurements (Figure 8) indicates melting of the probe tips. Hence, in some cases, the local temperature exceeds the melting point of tungsten $\left(3422^{\circ} \mathrm{C}\right)$, which is significantly above the sublima-



Figure 7: Scanning electron microscope image of the molten path due to the current flow between the contacts after a DC test showing evidence of melting and recrystallization of the material under electrical stress.

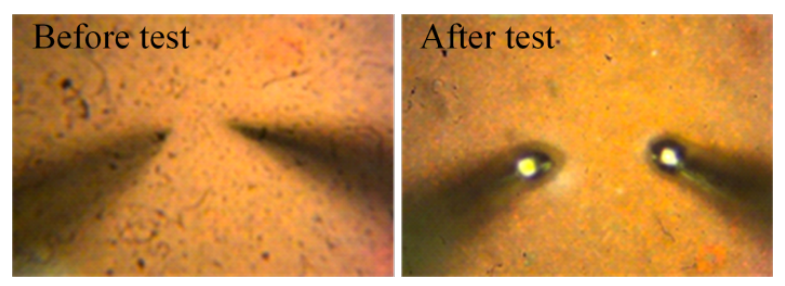

Figure 8: Optical microscope images showing two tungsten probes placed on a continuous $\mathrm{ZnO}$ nanoforest before (left) and after (right) a DC measurement test. The melting of the micrometer-sized probe tips during the electrical test suggests local temperatures above the melting point of tungsten $\left(3422^{\circ} \mathrm{C}\right)$.
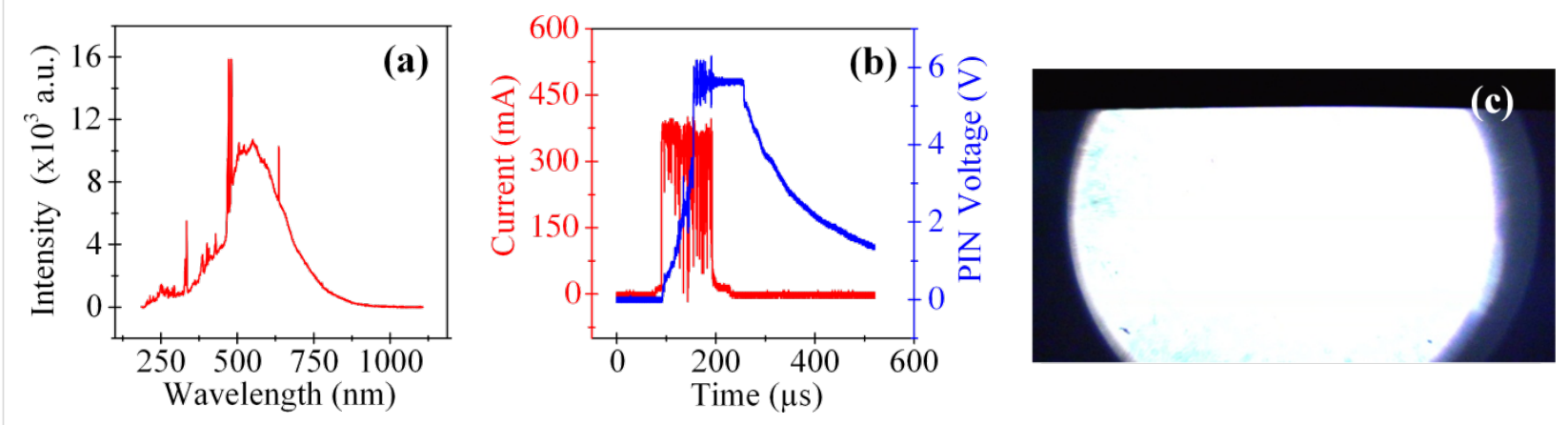

Figure 6: (a) Spectrum of the light emission resulting from application of a single pulse with amplitude of $45 \mathrm{~V}$ and duration of $100 \mu \mathrm{s}$. The spectral line around $480 \mathrm{~nm}$ was clipped due to the saturation of the spectrum analyzer. The white light (broad spectrum) content was very strong for this pulse. (b) Current and PIN output voltage versus time measured by an oscilloscope. The photodiode used for this trial was very sensitive but not fast enough to capture the details, showing slow rise and fall times and saturation. The delay was primarily due to the response time of the built-in amplifier in the PIN diode package. The fluctuations in the current indicate the recurrent formation and termination of conductive paths between the contacts. (c) A frame extracted from the corresponding light emission video. 
tion temperature of $\mathrm{ZnO}\left(\approx 380{ }^{\circ} \mathrm{C}\right.$ for the $\mathrm{Zn}$ surface, $\approx 600{ }^{\circ} \mathrm{C}$ for the $\mathrm{O}$ surface, and $\approx 1975^{\circ} \mathrm{C}$ for the decomposition into zinc vapor and oxygen) [17,25].

Figure 9a shows DC voltage sweep measurements performed in vacuum and at atmospheric pressure with a probe separation of $\approx 10 \mu \mathrm{m}$ on the $\mathrm{ZnO}$ nanorods grown on two adjacent polysilicon microstructures of very similar dimensions (length of $2.5 \mu \mathrm{m}$ and width of 2.1 and $2.15 \mu \mathrm{m}$ ). The resulting $I-V$ characteristics showed breakdown voltages at around 35 and $49 \mathrm{~V}$ at vacuum and atmospheric pressure, respectively. At lower pressure, lower temperature and hence, smaller applied electrical energy lead to sufficient vapor pressure to initiate the sublimation process [26].

The resulting light emission consisted of multiple flashes for the DC measurements both in vacuum and at atmospheric pressure. However, the light flashes in the atmospheric pressure measurements were more intense, whiter, and occurred over a broader area as compared to those in vacuum (Figure $9 \mathrm{~b}-\mathrm{g}$ ).

The observation of light emission in vacuum implies that breakdown in $\mathrm{ZnO}$, sublimation, and subsequent impact ionization are sufficient for the plasma formation process in the $\mathrm{ZnO}$ nanoforest, and hence, air breakdown is not required to initiate the plasma formation process.

\section{Conclusion}

The electrical experiments performed on $\mathrm{ZnO}$ nanorods grown on patterned silicon microstructures lead to high intensity, blue and white light emission. The abrupt increase in current at higher fields, sharp spectral peaks and very high local temperatures (observed as significant probe melting) indicate $\mathrm{ZnO}$ dielectric breakdown, sublimation, and plasma generation. The sharp spectral lines correspond to atomic electron transitions of excited $\mathrm{Zn}$ and $\mathrm{O}$ species in plasma and the spectra are similar to those from previous studies on $\mathrm{ZnO}$ light emission through the formation of a plasma plume [27,28]. The comparison between the results obtained from vacuum and atmospheric pressure measurements also indicate a plasma process as the dominant mechanism for light emission. The confinement of the plasma within a micro-chamber to contain the evaporated material [29] together with integrated electrodes may lead to a sustainable plasma state that could be tailored for various applications.

Even though many reports on light emission from $\mathrm{ZnO}$ refer to solid-state electroluminescence from homojunction or heterojunction structures, our results show that plasma formation can also take place with comparable electric fields and currents and can lead to blue and white light emission.

\section{Supporting Information}

Extracted frames from this video are shown in Figure 3.

\section{Supporting Information File 1}

High speed video of the light emission during DC test. [http://www.beilstein-journals.org/bjnano/content/ supplementary/2190-4286-6-255-S1.mov]

\section{Acknowledgements}

The authors are grateful to Kadir Cil and Lhacene Adnane (University of Connecticut) for their help with the electrical measurements and to Alan Lenef (Sylvania) for valuable discussions and the suggestion of the possibility of plasma formation. N. Noor and L. Lucera worked on the sample growth, physical characterization, electrical measurements and analysis and writing of the manuscript. L. Lucera was supported through the

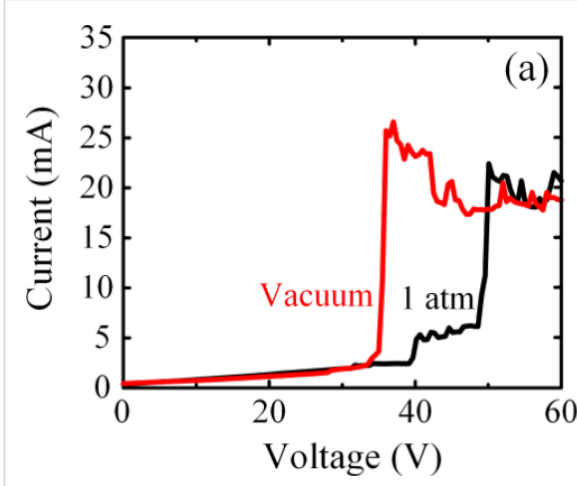

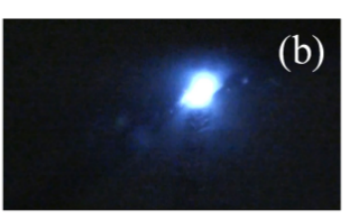

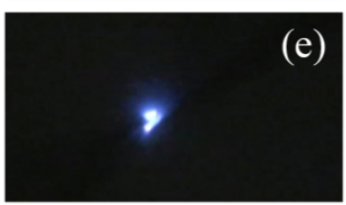

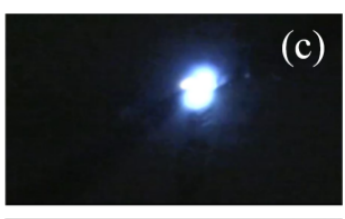

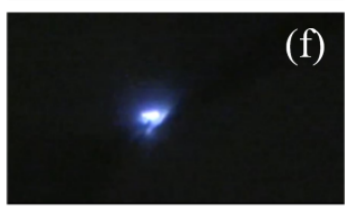

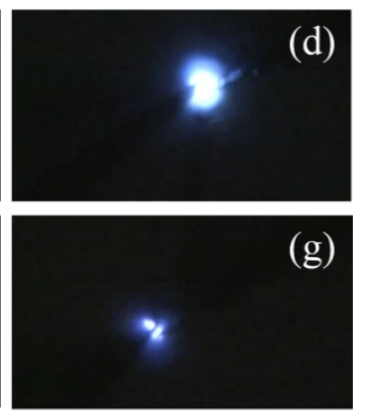

Figure 9: (a) $I-V$ characteristics for two DC voltage sweep measurements from 0-60 V performed in vacuum (red) and at atmospheric pressure (black). These measurements were performed on $\mathrm{ZnO}$ nanorods grown on two different polysilicon microstructures of very similar dimensions. (b-g) Video frames extracted from a high-resolution camera recording for the corresponding light emission for the DC measurements at atmospheric pressure $(b-d)$ and in vacuum $(e-g)$. 
EU-U.S. Dept. of Education Atlantis Program EAGLES (Engineers as Global Leaders for Energy Sustainability), application number USATD 18058. T. Capuano worked on improvement of the experimental setup and performed electrical measurements and was supported by the U.S. National Science Foundation through an REU supplement to Award ECCS 1150960. V. Manthina and A. Agrios worked on sample growth and physical analysis and were also supported by the U.S. National Science Foundation under Award CBET-1332022. H. Silva and A. Gokirmak worked on experiments, analysis and the manuscript preparation. N. Noor, H. Silva and A. Gokirmak were supported by the U. S. Department of Energy, Office of Basic Energy Sciences, Division of Materials Sciences and Engineering under Award DE-FG02-10ER46774.

\section{References}

1. Vinitha, B.; Manzoor, K.; Ajimsha, R.; Aneesh, P.; Jayaraj, M. Synth. React. Inorg., Met.-Org., Nano-Met. Chem. 2008, 38, 126-131.

2. Wang, Z. L. Mater. Today 2004, 7, 26-33. doi:10.1016/S1369-7021(04)00286-X

3. Zhang, L.; Jiang, Y.; Ding, Y.; Povey, M.; York, D. J. Nanopart. Res. 2007, 9, 479-489. doi:10.1007/s11051-006-9150-1

4. Bagnall, D. M.; Chen, Y. F.; Zhu, Z.; Yao, T.; Koyama, S.; Shen, M. Y.; Goto, T. Appl. Phys. Lett. 1997, 70, 2230-2232. doi:10.1063/1.118824

5. Purica, M.; Budianu, E.; Rusu, E. Thin Solid Films 2001, 383, 284-286. doi:10.1016/S0040-6090(00)01579-0

6. Hoffman, R. L.; Norris, B. J.; Wager, J. F. Appl. Phys. Lett. 2003, 82, 733-735. doi:10.1063/1.1542677

7. Lin, C. H.; Chiou, B.-S.; Chang, C. H.; Lin, J. D. Mater. Chem. Phys. 2003, 77, 647-654. doi:10.1016/S0254-0584(02)00120-7

8. Özgür, Ü.; Alivov, Y. I.; Liu, C.; Teke, A.; Reshchikov, M. A.; Doğan, S.; Avrutin, V.; Cho, S.-J.; Morkoç, H. J. Appl. Phys. 2005, 98, No. 041301. doi:10.1063/1.1992666

9. Ryu, Y.; Lee, T.-S.; Lubguban, J. A.; White, H. W.; Kim, B.-J.; Park, Y.-S.; Youn, C.-J. Appl. Phys. Lett. 2006, 88, 241108. doi:10.1063/1.2210452

10. Tsukazaki, A.; Kubota, M.; Ohtomo, A.; Onuma, T.; Ohtani, K.; Ohno, H.; Chichibu, S. F.; Kawasaki, M. Jpn. J. Appl. Phys. 2005, 44, L643. doi:10.1143/JJAP.44.L643

11. Guo, H.; Lin, Z.; Feng, Z.; Lin, L.; Zhou, J. J. Phys. Chem. C 2009, 113, 12546-12550. doi:10.1021/jp902607c

12. Baxter, J. B.; Aydil, E. S. Sol. Energy Mater. Sol. Cells 2006, 90 , 607-622. doi:10.1016/j.solmat.2005.05.010

13. Hsu, Y. F.; Xi, Y. Y.; Yip, C. T.; Djurišić, A. B.; Chan, W. K. J. Appl. Phys. 2008, 103, 083114. doi:10.1063/1.2909907

14. Ozgur, U.; Hofstetter, D.; Morkoç, H. Proc. IEEE 2010, 98, 1255-1268. doi:10.1109/JPROC.2010.2044550

15. Allan, G.; Bastard, G.; Boccara, N.; Lannoo, M.; Voos, M., Eds. Heterojunctions and Semiconductor Superlattices, Proceedings of the Winter School Les Houches, France, Les Houches, France, March 12-21, 1985; Springer Science \& Business Media, 2012.

16. Anthrop, D. F.; Searcy, A. W. J. Phys. Chem. 1964, 68, 2335-2342. doi:10.1021/j100790a052

17. Kohl, D.; Henzler, M.; Heiland, G. Surf. Sci. 1974, 41, 403-411. doi:10.1016/0039-6028(74)90057-0
18. Hari, P.; Baumer, M.; Tennyson, W. D.; Bumm, L. A. J. Non-Cryst. Solids 2008, 354, 2843-2848. doi:10.1016/j.jnoncrysol.2007.09.097

19. Wang, M.; Kim, E. J.; Hahn, S. H.; Park, C.; Koo, K.-K. Cryst. Growth Des. 2008, 8, 501-506. doi:10.1021/cg070467m

20. Shante, V. K. S. Phys. Rev. B: Condens. Matter Mater. Phys. 1977, 16, 2597. doi:10.1103/PhysRevB.16.2597

21. Ryu, Y.; Lee, T.-S.; Lubguban, J. A.; White, H. W.; Kim, B.-J.; Park, Y.-S.; Youn, C.-J. Appl. Phys. Lett. 2006, 88, No. 241108.

22. Zhang, X.-M.; Lu, M.-Y.; Zhang, Y.; Chen, L.-J.; Wang, Z. L. Adv. Mater. 2009, 21, 2767-2770. doi:10.1002/adma.200802686

23. Baltakesmez, A.; Tekmen, S.; Tüzemen, S. J. Appl. Phys. 2011, 110, 054502. doi:10.1063/1.3627247

24. Willander, M.; Nur, O.; Sadaf, J. R.; Qadir, M. I.; Zaman, S.; Zainelabdin, A.; Bano, N.; Hussain, I. Materials 2010, 3, 2643-2667. doi:10.3390/ma3042643

25. Ellmer, K.; Klein, A.; Rech, B. Transparent Conductive Zinc Oxide: Basics and Applications in Thin Film Solar Cells; Springer: Berlin, Germany, 2007.

26. Pavia, D.; Kriz, G.; Lampman, G.; Engel, R. A Microscale Approach to Organic Laboratory Techniques; Cengage Learning, 2012.

27. Hanif, M.; Salik, M.; Baig, M. A. Plasma Chem. Plasma Process. 2013, 33, 1167-1178. doi:10.1007/s11090-013-9478-0

28. Saji, K. J.; Joshy, N. V.; Jayaraj, M. K. J. Appl. Phys. 2006, 100, 043302. doi:10.1063/1.2266260

29. Eden, J. G.; Park, S.-J. Plasma Phys. Controlled Fusion 2005, 47, B83. doi:10.1088/0741-3335/47/12B/S07

\section{License and Terms}

This is an Open Access article under the terms of the Creative Commons Attribution License (http://creativecommons.org/licenses/by/2.0), which permits unrestricted use, distribution, and reproduction in any medium, provided the original work is properly cited.

The license is subject to the Beilstein Journal of Nanotechnology terms and conditions: (http://www.beilstein-journals.org/bjnano)

The definitive version of this article is the electronic one which can be found at: $\underline{\text { doi:10.3762/bjnano. } 6.255}$ 